\title{
ON CERTAIN TYPES OF PLANE CONTINUA*
}

BY

\author{
N. E. RUTT $†$
}

Continua which are the sum of a set of continua mutually exclusive except for a point which all have in common play a rôle of importance in analysis situs. In particular they are likely to obtrude upon any study of unbounded point sets, since in connection with these it is so often of value to perform an inversion of space. An investigation of some properties of such continua is the task of this paper. In it will be derived some interrelations of the almost mutually exclusive continua out of which they are built.

1. Introduction. A property of upper semicontinuous collections of continuał presently to be found useful in several connections will be derived first.

Lemma I. If $S$ is a euclidean plane, $G$ is an upper semicontinuous collection of mutually exclusive bounded continua filling up $S$ and none dividing $S, K$ is a bounded continuous curve contained in $S$, and $G_{k}$ is that maximal\$ subcollection of $G$ each of whose elements contains a point of $K$, then $G_{k}$ is a bounded continuous curve of elements of $G$. $\mid$

Lemma II. If $S$ is a euclidean plane, $G$ and $H$ are upper semicontinuous collections of mutually exclusive bounded continua filling up $S$ and none dividing it, and every element of $H$ is a subset of some element of $G$, then in the space $W$ whose points are the elements of $H$ the point sets corresponding to the elements of $G$ are an upper semicontinuous collection of mutually exclusive bounded continua filling $u p W$ and none dividing $W$.

THEOREM I. II If $S$ is a euclidean plane, $G$ is an upper semicontinuous collection of mutually exclusive bounded continua filling up $S$ and none dividing it, $M$, and $N_{8}$ are two simple closed curves of $S$ whose points are elements of $G$, and

* Presented to the Society, June 21, 1929; received by the editors July 28, 1930.

$\dagger$ National Research Fellow, University of Texas, Austin, Texas.

$\ddagger$ For definitions and development of fundamental properties of such collections, see R. L. Moore, Concerning upper semi-continuous collections of continua, these Transactions, vol. 27 (1925), pp. 416428. Moore proves if the elements of an upper semicontinuous collection of mutually exclusive bounded continua filling a euclidean plane and none dividing it be considered the points of a space, that this space is itself topologically equivalent to a euclidean plane.

$\S$ Maximal in the sense that it is a proper subset of no similarly defined subcollection of $G$.

T For proof of this see H. M. Gehman, A special type of upper semicontinuous collection, Proceedings of the National Academy of Sciences, vol. 16, No. 9, pp. 609-613, Theorem III.

$\|$ The writer is indebted to Professor H. M. Gehman for a suggestion which has given this theorem a more general form than that which the writer obtained originally. 
$a_{s}^{m}, b_{s}^{m}$, and $c_{s}^{m}$ are distinct points of $M_{s}$ while $a_{s}^{n}, b_{s}^{n}$, and $c_{s}^{n}$ are distinct points of $N_{s}$; then $a_{s}^{m}, b_{s}^{m}$, and $c_{s}^{m}$ have the same sense on $M_{s}$ as $a_{s}^{n}, b_{s}^{n}$, and $c_{s}^{n}$ have on $N_{s}$ if and only if the corresponding points $a_{t}^{m}, b_{t}^{m}$, and $c_{t}^{m}$ on the corresponding curve $M_{t}$ in the space $T$ whose points are the elements of $G$ have the same sense as $a_{t}^{n}$, $b_{t}^{n}$, and $c_{t}^{n}$ upon $N_{t}{ }^{*}$

An outline of the proof of this theorem will be sufficient.

Suppose first that there exists a simple closed curve $L_{s}$ which together with its interior is contained in the common exterior of $M_{s}$ and $N_{s}$ and which also is composed of points that are themselves elements of $G$.

Let $a_{s}^{l}, b_{s}^{l}$, and $c_{s}^{l}$ and $a_{t}^{l}, b_{t}^{l}$, and $c_{l}^{l}$ be corresponding sets of distinct points upon the corresponding curves $L_{s}$ and $L_{t}$ respectively. It will be considered that the assertion is established for the special case now being considered when it has been shown that if $a_{s}^{m}, b_{s}^{m}$, and $c_{s}^{m}$ may be simply joined to $a_{s}^{l}, b_{s}^{l}$, and $c^{l}$, then $a_{t}^{m}, b_{t}^{m}$, and $c_{t}^{m}$ may be simply joined to $a_{t}^{l}, b_{t}^{l}$, and $c_{l}^{l}$, and conversely.

Suppose that $a_{s}^{m}, b_{s}^{m}$, and $c_{s}^{m}$ may be simply joined to $a_{s}^{l}, b_{s}^{l}$, and $c_{s}^{l}$. Let $A_{s}$ be an arc joining $a_{s}^{m}$ and $a_{s}^{l}$ in the common exterior of $L_{s}$ and $M_{s}$, and let $G_{a}$ be the maximal subaggregate of $G$ each element of which contains a point of $A_{s}$. Owing to Lemma I, $G_{a}$ contains as a subset an arc of elements $H_{a}$ from $a_{s}^{m}$ to $a_{s}^{l}$ which as a subset of $S$ is a continuum $K_{a}$ not dividing the plane and contained except for the points $a_{s}^{m}$ and $a_{s}^{l}$ in the common exterior of $L_{s}$ and $M_{s}$. Clearly there may now be constructed in the common exterior of $L_{s}$ and $M_{s}$ and the complement of $K_{a}$ an arc $B_{s}$ with end points $b_{s}^{m}$ and $b_{s}^{l}$. The arc $B_{s}$, like $A_{s}$ above, identifies a subcollection $G_{b}$ of $G$ including an arc of elements $H_{b}$ of $G$ whose one end element is $b_{s}^{m}$ and the other $b_{s}^{l}$, and whose sum is a continuum $K_{b}$ contained except for the points $b_{s}^{m}$ and $b_{s}^{l}$ in the common exterior of $L_{s}$ and $M_{s}$ and the complement of $K_{a}$, and not dividing space. Let $M$ and $L$ be arcs with end points respectively $a_{s}^{m}, b_{s}^{m}$ and $a_{s}^{l}, b_{s}^{l}$ contained except for their ends in the interiors of the simple closed curves $M_{s}$ and $L_{s}$. Then $L+M+A_{s}+B_{s}$ is a simple closed curve $X$ and $L+M+K_{a}+K_{b}$ is a continuum $Y$ which divides $S$ into precisely two mutually separated connected sets. If $Y$ were to separate $c_{s}^{m}$ from $c_{s}^{l}$ it would follow that $X$ must do this also, a contradiction of hypothesis, so there exists in the common exterior of $L_{s}$ and $M_{s}$ and in the complement of $Y$ an $\operatorname{arc} C_{s}$ with end points $c_{s}^{m}$ and $c_{s}^{l}$. This arc identifies a subcollection $G_{c}$ of $G$ including an arc of elements $H_{c}$ of $G$ whose

* For proofs and discussion of the fundamental problems of sense underlying this and similar statements, see J. R. Kline, $A$ definition of sense on closed curves in non-metrical plane analysis situs, Annals of Mathematics, (2), vol. 19 (1917-1918), pp. 185-200; also by the same author, Concerning sense on closed curves in non-metrical analysis situs, Annals of Mathematics, (2), vol. 21 (1919-1920), pp. 113-119. 
one end element is $c_{s}^{m}$ and the other $c_{s}^{\prime}$, and whose sum is a continuum $K_{c}$ contained except for the points $c_{s}^{m}$ and $c_{s}^{l}$ in the common exterior of $L_{s}$ and $M_{s}$ and the complement of $Y$. Thus $H_{a}, H_{b}$, and $H_{c}$ correspond in $T$ to the three $\operatorname{arcs} A_{t}, B_{t}$, and $C_{t}$ simply joining $a_{t}^{m}, b_{t}^{m}$, and $c_{t}^{m}$ to $a_{t}^{l}, b_{t}^{l}$, and $c_{l}^{l}$.

Suppose conversely that $A_{t}, B_{t}$, and $C_{t}$ are three arcs simply joining $a_{t}^{m}$, $b_{t}^{m}$, and $c_{l}^{m}$ to $a_{t}^{l}, b_{t}^{l}$, and $c_{t}^{l}$. In $S$ and corresponding to these are three continua $K_{a}, K_{b}$, and $K_{c}$ mutually exclusive and contained, except for the respective pairs of points $a_{s}^{m}$ and $a_{s}^{l}, b_{s}^{m}$ and $b_{s}^{l}, c_{s}^{m}$ and $c_{s}^{l}$, in the common exterior of $M_{s}$ and $L_{s}$. Neighborhoods of $K_{a}, K_{b}$, and $K_{c}$ exist in which it may readily be seen that three arcs $A_{s}, B_{s}$, and $C_{s}$ may be drawn simply joining $a_{s}^{m}, b_{s}^{m}$, and $c_{s}^{m}$ to $a_{s}^{l}, b_{s}^{l}$, and $c_{s}^{l}$.

Suppose now that there exists no simple closed curve $L_{s}$ satisf ying the conditions mentioned above. Let $\sigma_{s}$ be a bounded connected and simply connected subdomain of elements of $G$ containing $M_{s}+N_{s}$. Let $\rho_{s}$ be a bounded connected and simply connected subdomain of elements of $G-\sigma_{s}$. Let $H$ be the collection whose elements are the elements of $G$ in $\bar{\rho}_{s}$ and all points of $S$ not contained in any element of $G$ belonging to $\bar{\rho}_{s}$. In $W$, the space whose points are the elements of $H$, let $L_{w}$ be a simple closed curve contained in $\rho_{w}$. Now $a_{s}^{n}, b_{s}^{m}$, and $c_{s}^{m}$ can be simply joined to $a_{s}^{n}, b_{s}^{n}$, and $c_{s}^{n}$ if and only if $a_{w}^{m}, b_{w}^{m}$, and $c_{w}^{m}$ can be simply joined to $a_{w}^{n}, b_{w}^{n}$, and $c_{w}^{n}$, for $S$ and $W$ within $\sigma_{s}$ and $\sigma_{w}$ are in continuous one to one reciprocal correspondence; and $a_{w}{ }_{w}$, $b_{w}^{m}$, and $c_{w}^{m}$ can be simply joined to $a_{w}^{l}, b_{w}^{l}$, and $c_{w}^{l}$ if and only if $a_{t}^{m}, b_{t}^{m}$, and $c_{t}^{m}$ can be simply joined to $a_{t}^{l}, b_{t}^{l}$, and $c_{t}^{l}$, by the first part of the argument. These two facts together with Lemma II permit the desired conclusion to be deduced easily.

CoRollaRy I. If $S$ is a euclidean plane, $G$ is an upper semicontinuous collection of mutually exclusive bounded continua filling up $S$ and none dividing it, $M_{8}$ and $N_{8}$ are two simple closed curves of $S$ and all elements of $G$ containing points of $M_{s}$ or $N_{s}$ are respectively subsets of $M_{s}$ or $N_{s}$, and $A_{s}^{m}, B_{s}^{m}$, and $C_{s}^{m}$ are distinct elements of $G$ contained by $M_{s}$ while $A_{s}^{n}, B_{s}^{n}$, and $C_{s}^{n}$ are distinct elements of $G$ contained by $N_{s} ;$ then $A_{s}^{m}, B_{s}^{m}$, and $C_{s}^{m}$ have the same sense on $M_{s}$ as $A_{s}^{n}, B_{s}^{n}$, and $C_{s}^{n}$ have on $N_{s}$ if and only if the corresponding points $a_{t}^{m}, b_{t}^{m}$, and $C_{t}{ }^{m}$ on the corresponding curve $M_{t}$ in the space $T$ whose points are the elements of $G$ have the same sense as $a_{t}^{n}, b_{t}^{n}$ and $c_{t}^{n}$ upon $N_{t}{ }^{*}$

The corollary follows readily from Lemma II and Theorem I.

\footnotetext{
${ }^{*}$ Let $a_{s}^{m}, b_{s}^{m}, c_{s}^{m}$ and $a_{s}{ }^{n}, b_{s}{ }^{n}, c_{s}{ }^{n}$ be points contained respectively by the continua $A_{s}^{m}, B_{s}^{m}, C_{s}^{m}$ and $A_{s}{ }^{n}, B_{s}{ }^{n}, C_{s}{ }^{n}$. Then the sense of $A_{s}^{m}, B_{s}^{m}, C_{s}^{m}$ is said to be the same as that of $A_{s}{ }^{n}, B_{s}{ }^{n}, C_{s}{ }^{n}$ on $M_{s}$ and $N_{s}$ respectively, or different from it, if the sense of $a_{s}^{m}, b_{s}^{n}, c_{s}^{m}$ is the same as that of $a_{s}{ }^{n}, b_{s}{ }^{n}, c_{s}{ }^{n}$ or different from it.
} 
2. Notation. The letter $Z$ will be used to designate a plane bounded point set composed of the continuum $X$ and the set of continua $\left[X_{\alpha}\right]$, where each element of $\left[X_{\alpha}\right]$ contains a point of $X$, no two have any point in common which is not a point of $X$, no one is disconnected by the omission from it of its subset in $X$, and no one when added to $X$ forms a point set any bounded component of whose complement contains any point whatever of an element of $\left[X_{\alpha}\right]$.

If $C$ is a simple closed curve in $S$, let $i(C)$ represent its bounded complementary domain or interior, and $e(C)$ its unbounded complementary domain or exterior. If $K$ is a point set in $S$ let $c(K)$ be the subset of $S$ complementary to $K$. Thus, in $S, c i(C)$ represents the complement of the interior of the simple closed curve $C$, that is, $C+e(C)$, and $c e(C)$ the complement of its exterior, that is, $C+i(C)$.

Remarks. Order may be assigned among the elements of $\left[X_{\alpha}\right]$. A description of one method for doing this is already in print.* Another may be outlined as follows. If $C$ is any simple closed curve enclosing $X$, and $X_{a}$ is any element of $\left[X_{\alpha}\right]$, let $X_{a}^{c}$ be the sum of the components of $X_{a} \cdot i(C)$ each of which contains a point of $X$. Associated with each element $X_{a}^{c}$ of $\left[X_{\alpha}\right]$ is a subarc $C_{a}$ of $C$ containing all the points of $\bar{X}_{a}^{c} \cdot C$ and containing no point whatever in common with the subarc of $C$ similarly associated with any other element of $\left[X_{\alpha}\right]$. Suppose that both $C$ and $D$ are simple closed curves which enclose $X$ and which intersect each of the three different elements $X_{l}, X_{m}$, and $X_{n}$ of $\left[X_{\alpha}\right]$. Then the sense of $C_{l}, C_{m}$, and $C_{n}$ upon $C$ is the same as the sense of $D_{l}, D_{m}$, and $D_{n}$ upon $D$. Accordingly no confusion can arise if the sense of those elements $\left[X_{\alpha}\right]_{c}$ of $\left[X_{\alpha}\right]$ having non-vacuous intersections with the simple closed curve $C$ be defined as the same as the sense of the non-vacuous elements of $\left[C_{\alpha}\right]$. Of course the set $\left[X_{\alpha}\right]_{c}$ may be not identical with $\left[X_{\alpha}\right]$; however, it is clear that by selecting a simple closed curve properly the order relations of any particular element of $\left[X_{\alpha}\right]$ not included in $\left[X_{\alpha}\right]_{c}$ with the elements of $\left[X_{\alpha}\right]_{c}$ may be determined. It is evident now what will be meant by the separation of two elements of $\left[X_{\alpha}\right]$ by some other pair of them. With reference to some particular element $X_{r}$ of $\left[X_{\alpha}\right]$ the expressions $X_{a}$ precedes $X_{b},\left[X_{i}\right](i=1,2,3, \cdots)$ is a clockwise series and $\left[X_{i}\right]$ is a counterclockwise series, all of course referring to the elements of $\left[X_{\alpha}\right]$, may be defined. When, for instance, $X_{i}$ precedes $X_{i+1}(i=1,2,3, \cdots)$ in the series $\left[X_{i}\right]$, the series will be called clockwise. In what follows it is immaterial which sense about the simple closed curve $C$ is referred to as clockwise, but a

* R. L. Moore, Concerning the sum of a countable number of continua in the plane, Fundamenta Mathematicae, vol. 6, pp. 189-2(12. 
definite one of the two is of course assumed to be such at the outset. It will be convenient to assume that $X_{r}-X$ contains a point which is the end of a ray $R$, all points of which except its end point belong to the complement of $Z$. It proves unnecessary to specify whether $X_{r}$ precedes or follows other elements of $\left[X_{\alpha}\right]$. If $a b$ is an arc with its ends, $a$ and $b$ respectively, in $X_{a}-X_{a} \cdot X$ and $X_{b}-X_{b} \cdot X$ and no other points whatever in $X_{a}+X_{b}+X_{r}+X+R$, then there exists a bounded complementary domain $\delta$ of $X_{a}+X_{b}+X+a b$ such that if $T$ represents the sum of the components of $X_{c} \cdot c(a b+X)$ with limit points in $X$, then $\delta$ contains $T$ or contains no points of $T$ according as the element $X_{c}$ of $\left[X_{\alpha}\right]$ is or is not between $X_{a}$ and $X_{b}$.

Lemma III. Given (1) $Z$ is the set defined above and $X$ is a point; (2) $a$ is a point of $Z-X$ and $X_{a}$ is the element of $\left[X_{\alpha}\right]$ containing $a$; (3) $X_{1}, X_{2}, \cdots$ is a clockwise series of elements of $\left[X_{\alpha}\right]$ all following $X_{a}$ and such that $a$ is a limit point of $\sum X_{i}$; (4) $V$ is a ray in $c\left(X_{a}\right)$ containing a point of $X_{1}$. Then $V$ contains a point of at least one other element of the series.

Let $v$ be the end of $V$ and $v^{*}$ be the last point of $V$ belonging to $X_{1}$. The lemma will be proved about $V^{*}$, that subray of $V$ whose end is $v^{*}$. Suppose that the lemma is false and that for every subscript $n, n>1, V^{*} \cdot X_{n}=0$. Let $\left[C_{i}\right]$ be a set of circles having common center $a$, satisfying for $i=1,2,3, \cdots$ the condition $i\left(C_{i}\right) \supset c e\left(C_{i+1}\right)$, and having radii converging to zero. Let the radius of $C_{1}$ be less than $d\left(a, X+X_{1}+X_{r}+R\right)$. As $a$ is a limit point of $\sum X_{i}$ there is a first element $X_{1}^{*}$ of $\left[X_{i}\right]$ following $X_{1}$ containing a point of $c e\left(C_{1}\right)$, and a first circle $C_{2}^{*}$ of $\left[C_{i}\right]$ containing no point of $X_{1}^{*}$. As $a$ is a limit point of $\sum X_{i}$ there is a first element $X_{2}^{*}$ of $\left[X_{i}\right]$ containing a point of $c e\left(C_{2}^{*}\right)$, and a first circle $C_{3}^{*}$ of $\left[C_{i}\right]$ containing no point of $X_{2}^{*}$. In general as $a$ is a limit point of $\sum X_{i}$ there is a first element $X_{j}^{*}$ of $\left[X_{i}\right]$ containing a point of $c e\left(C_{j}^{*}\right)$, and a first circle $C_{j+1}^{*}$ of $\left[C_{i}\right]$ containing no point of $X_{j}^{*}$. The infinite sequences $\left[X_{i}^{*}\right]$ and $\left[C_{i}^{*}\right]$ satisfy all the hypotheses imposed by supposition upon $\left[X_{i}\right]$ and $\left[C_{i}\right]$. Obviously $V^{*}$ has no point in any element of $\left[X_{i}^{*}\right]$. For $i=1,2,3, \cdots$ let $S_{i}$ be a straight line segment contained in $c e\left(C_{i}^{*}\right)$ with one end in $X_{a}$, the other in $X_{i}^{*}$, and no additional point whatever in $X_{a}+X_{i}^{*}$. As $S_{i} \cdot\left(X_{r}+R\right)=0$, by reason of a remark made above, $S_{i}+X_{a}+X_{i}^{*}$ for each value of $i$ bounds a bounded complementary domain $\delta_{i}$ which must contain all but the point $X$ of $X_{1}$. The domain $\delta_{i}$ contains points of $V^{*}$ as it contains $v^{*}$, but can not contain $V^{*}$ as $v^{*}$ is unbounded. Thus for each value of $i$ the boundary $\Delta_{i}$ of $\delta_{i}$ contains a point of $V^{*}$. Since $\Delta_{i} \subset S_{i}+X_{a}+X_{i}^{*}$ and by assumption $V^{*} \cdot\left(X_{i}^{*}+X_{a}\right)=0, V^{*} \cdot S_{i} \neq 0$ for each value of $i$. The set of points $\sum V^{*} \cdot S_{i}$ being contained in $V^{*}$ has all its limit points in $V^{*}$. As it has a pcint within any circle whose center is $a$, it contains $a$ or has $a$ as limit point. In either case $V^{*}$ contains $a$, a contradiction. The lemma is thus established. 
THEOREM II. Suppose that $X$ is a point and $Z$ a continuum. If $a$ is any point of $Z-X$ and $X_{a}$ is the element of $\left[X_{\alpha}\right]$ containing $a$, and $\left[X_{i}\right](i=1,2,3, \cdots)$ is a clockwise series of elements of $\left[X_{\alpha}\right]$ all following $X_{a}$, then a is not a limit point of $\sum X_{i}$.

Let $C$ be a circle whose interior contains $Z$. Let $\left[Y_{\alpha}\right]$ be the subset of continua of $\left[X_{\alpha}\right]$ consisting of those each of which contains a point different from $X$ which may be joined to a point of $C$ by an arc contained except for one end in the complement of $Z$. Let $A_{p}$ be any such arc associated with $Y_{p}$.

Let the elements of $\left[X_{\alpha}\right]$ be well ordered. $\dagger$ This having been done the elements of $\left[Y_{\alpha}\right]$ are also well ordered. Let $Y_{1}^{1}, Y_{2}^{1}, Y_{3}^{1}$, and $Y_{4}^{1}$ be the first four elements of $\left[Y_{\alpha}\right]$, and let no two of the $\operatorname{arcs} A_{1}, A_{2}, A_{3}$, and $A_{4}$ have a common point and no one have more than one point in $C$. Let the four sets $Y_{1}^{1}, Y_{2}^{1}, Y_{3}^{1}$, and $Y_{4}^{1}$ be grouped in pairs each of which separates the other in $\left[X_{\alpha}\right]$. Suppose that $Y_{i}^{1}$ and $Y_{j}^{1}$ separate $Y_{p}^{1}$ and $Y_{q}^{1}, i, j, p$, and $q$ being 1 , 2,3 , and 4 in some order. Then $i(C) \cdot\left(Y_{i}{ }^{1}+A_{i}{ }^{1}+Y_{j}^{1}+A_{j}{ }^{1}\right)$ separates $i(C)$ into two domains each containing a subset of $Z$, these being respectively $z_{p}^{1}$ and $z_{q}^{1}$. Let $Z_{p}^{1}=z_{p}{ }^{1}+Y_{i}{ }^{1}+Y_{j}{ }^{1}$ and $Z_{q}{ }^{1}=z_{q}{ }^{1}+Y_{i}{ }^{1}+Y_{j}{ }^{1}$. Both $Z_{p}{ }^{1}$ and $Z_{q}^{1}$ are continua similar in structure to the continuum $Z$.

Now suppose that the theorem is not true. Then $\left[X_{\alpha}\right]$ contains an element $X_{a}$ containing a point $a$ different from $X$, and a series $\left[X_{i}\right]$ clockwise and including only elements of $\left[X_{\alpha}\right]$ which follow $X_{a}$, such that the point set $\sum X_{i}$ has $a$ among its limits. From Lemma III it follows that the pair of elements $Y_{i}^{1}$ and $Y_{j}^{1}$ can not separate two elements of $\left[X_{i}\right]$, for if they could, a proper selection of one of them as $X_{r}$ and the other as $X_{1}$ would contradict the lemma, as both, owing to the existence of $A_{i}^{1}$ and $A_{j}^{1}$, contain points different from $X$ which are ends of rays contained except for their ends in the complement of $Z$. Accordingly the elements of $\left[X_{i}\right]$ can not be distributed between $Z_{p}^{1}$ and $Z_{q}^{1}$ but must occur all in one of the two, say in $Z_{p}^{1}$. Since $Z_{p}^{1}$ is a continuum and $a$ is a limit of the set $\sum X_{i}, Z_{p}^{1}$ contains points of $X_{a}$ and must therefore contain $X_{a}$. It thus appears that $Z_{p}^{1}$ like $Z$ is a continuum about which the theorem is not true. Let $Z_{p}^{1}$ be $Z^{1},\left[X_{\alpha}^{1}\right]$ be the subset of elements of $\left[X_{\alpha}\right]$ each of which contains a point different from $X$ in $Z^{1}$ and so is entirely contained in $Z^{1}$, and $\left[Y_{\alpha}^{1}\right]$ be the subset of the elements of $\left[X_{\alpha}^{1}\right]$ each of which contains a point different from $X$ arcwise accessible from $C$ in the complement of $Z^{1}$. It is readily seen that both $\left[X_{\alpha}^{1}\right]$ and $\left[Y_{\alpha}^{1}\right]$ are well ordered and that $\left[Y_{\alpha}^{1}\right] \subset\left[Y_{\alpha}\right]$.

With $n=2,3,4, \cdots$, let $Y_{1}^{n}, Y_{2}, Y_{3}^{n}$, and $Y_{4}^{n}$ be the first four elements of $\left[Y_{\alpha}^{n-1}\right]$. At least one of these follows all four of $\left[Y_{k}^{n-1}\right](k=1,2,3,4)$ in the

$\dagger$ It is well known that this may be accomplished by means of the Zermelo postulate. 
well ordered sequence $\left[Y_{\alpha}^{n-1}\right]$. The $\operatorname{arcs} A_{1}^{n}, A_{2}^{n}, A_{3}^{n}$, and $A_{4}^{n}$ exist as in the first case. Suppose that $Y_{i}^{n}$ and $Y_{j}^{n}$ separate $Y_{p}^{n}$ and $Y_{q}^{n}$ in $\left[X_{\alpha}^{n-1}\right]$. Then $i(C) \cdot\left(Y_{i}^{n}+A_{i}^{n}+Y_{j}^{n}+A^{n}\right)$ separates $i(C)$ into two domains each containing a subset of $Z, z_{p}^{n}$ and $z_{q}^{n}$ respectively. Let $Z_{p}^{n}=z_{p}^{n}+Y_{i}^{n}+Y_{j}^{n}$, and $Z_{q}^{n}=z_{q}^{n}$ $+Y_{i}^{n}+Y_{j}^{n}$. As in the case for $n=1$, these are continua like $Z$. One of them, say $Z_{p}^{n}$, contains $X_{a}$ and $\left[X_{i}\right]$, and consists of a well ordered subset [ $\left.X_{\alpha}^{n}\right]$ of the elements of $\left[X_{\alpha}\right]$. Evidently the subset $\left[Y_{\alpha}^{n}\right]$ may be defined as $\left[Y_{\alpha}^{1}\right]$ was defined, is then well ordered, and is a subset of $\left[Y_{\alpha}^{n-1}\right]$.

For each value of $i(i=1,2,3, \cdots)$ there is thus determined a continuum $Z^{i}$, no two identical, which is of the same type as $Z$, and contains both $X_{a}$ and the series $\left[X_{i}\right]$. Each of $\left[Z^{i}\right]$ is composed of a certain subset of the elements of $\left[X_{\alpha}\right]$. Taken together they form a well ordered sequence. Let

$$
Z^{\omega}=\prod_{i=1}^{\infty} Z^{i}
$$

Then $Z^{\omega} \supset X$, and if $Z^{\omega} \supset p$ where $p$ is a point not $X$ of $X_{p}$, an element of $\left[X_{\alpha}\right]$, then $Z^{\omega} \supset X_{p}$. Therefore $Z^{\omega}$ is a continuum of the same kind as $Z$. Let it be made up of the elements $\left[X_{\alpha}^{\omega}\right]$ of $\left[X_{\alpha}\right]$ and let $\left[Y_{\alpha}^{\omega}\right]$ be the subset of these whose relation to $C$ and the complement of $Z^{\omega}$ is like the relation of the elements of $\left[Y_{\alpha}\right]$ to $C$ and the complement of $Z$. Clearly $\left[Y_{\alpha}^{\omega}\right]$ is a well ordered aggregate. It is not true in general that $\left[Y_{\alpha}^{\omega}\right] \subset\left[Y_{\alpha}^{i}\right]$, but it is true that

$$
\left[Y_{\alpha}^{\omega}\right] \supset \prod_{i=1}^{\infty}\left[Y_{\alpha}^{i}\right]
$$

Evidently $Z^{\omega} \supset\left(X_{a}+\sum X_{i}\right)$.

It is now clear how to extend the well ordered sequence $\left[Z^{\alpha}\right]$. If at any stage this sequence has a last element $Z^{f-1}$, the next may be defined by selecting $Y_{1}^{f}, Y_{2}^{f}, Y_{3}^{f}$, and $Y_{4}^{f}$, the first four elements of $\left[Y_{\alpha}^{f-1}\right]$, choosing two that separate the other two, and thus obtaining a proper subcontinuum $Z^{f}$ of $Z^{f-1}$ composed of elements of $\left[X_{\alpha}\right]$ and including $X_{a}$ and $\left[X_{i}\right]$. If at any stage the sequence has no last element, then the next element consists of the set of points common to all the elements of the sequence already defined, a continuum which clearly exists as it contains $X_{a}$ and $\left[X_{i}\right]$ and just as clearly consists of a subset of the elements of $\left[X_{\alpha}\right]$.

Under what conditions will it be impossible to extend the sequence $\left[Z^{\alpha}\right]$ ? If this does become impossible clearly that must be in some case where the sequence up to that point defined has a last element, as when it has no last element the process being employed immediately determines another, namely the set of points common to all the elements already determined. Suppose then that $Z^{\lambda}$ is the last element of $\left[Z^{\alpha}\right]$. Can $Z^{\lambda}$ contain four or more ele- 
ments of $\left[X_{\alpha}\right]$ ? Supposing that it can, various simple methods for proving that the subset $\left[Y_{\alpha}^{\lambda+1}\right]$ of $\left[X_{\alpha}\right]$ consists of at least four elements will soon present themselves. Such being the case it would be possible immediately to obtain an element following $Z^{\lambda}$, and so $Z^{\lambda}$, the last element of $\left[Z^{\alpha}\right]$, is composed of fewer than four of the elements of $\left[X^{\alpha}\right]$.

But the assumption that the theorem was not true of the continuum $Z$ in the particular instance of $X_{a}$ and $\left[X_{i}\right]$ has been seen to imply that each element of $\left[Z^{\alpha}\right]$ contains $X_{a}+\sum X_{i}$. Thus $Z^{\lambda}$ must contain more than four elements of $\left[X_{\alpha}\right]$. The contradiction between these two inferences concerning $Z^{\lambda}$ establishes the theorem.

Corollary II. Suppose $Z$ is a continuum. If $a$ is a point of $Z-X$ and $X_{a}$ is the element of $\left[X_{\alpha}\right]$ containing $a$, and $\left[X_{i}\right](i=1,2,3, \cdots)$ is a clockwise series of the elements of $\left[X_{\alpha}\right]$ all following $X_{a}$, then a is not a limit of $\sum X_{i}$.

Let $D$ be the sum of the bounded components of $c(X) . X+D$ is a continuum $K$. Let $G$ be the upper semicontinuous collection of mutually exclusive bounded continua whose elements are respectively $K$ and the points of $c(K)$. The elements of $G$ are points of a space $T$ which is topologically equivalent to $S$. $\dagger$ In $T$ there corresponds to the continuum $Z$ of $S$ a continuum $Z_{t}$ of the same type as $Z$ except that the set $X_{t}$ corresponding to $X$ is a single point. Concerning $Z_{t}$, accordingly, Corollary II is identical with Theorem II and is therefore true. But if it were to be supposed that Corollary II is not true of $Z$, then owing to Corollary I and the nature of $Z_{t}$ it would follow that Corollary II could not be true for $Z_{t}$ either; a contradiction.

3. Notation. In the following paragraphs capital letters in script, as $\mathbb{P}$, will represent prime ends.

Remarks. It will be assumed in this paper that the prime ends discussed are defined by chains of cuts whose members are simple continuous arcs. Some facts which may easily be deduced from published researches upon prime ends and which will be used presently are as follows. Any connected and simply connected domain whose boundary is bounded, although the domain may itself be unbounded, may have its prime ends defined in the usual way, and these may then be shown to have cyclic arrangement. $\$$

Suppose that $X$ is a single point. If $X_{a}$ is any element of $\left[X_{\alpha}\right]$, then $X$ is arcwise accessible from one and but one prime end of the boundary of the

\footnotetext{
$\dagger$ K. L. Noore, Concerning.upper semiciontinuous collections of continua, loc. cit., p. 424, Theorem 21.

$\ddagger$ For definitions and fundamental research upon prime ends, see C. Carathéodory, Über die Begrensung einfach susummenhängender Gebiete, Mathematische Annalen, vol.73 (1912), pp. 323-370.

$\S$ In the paper just referred to, Carathéodory considers only bounded domains.
} 
unbounded component of $c\left(X_{a}\right)$. If $X_{a}$ and $X_{b}$ are distinct elements of [ $\left.X_{\alpha}\right]$ then $X$ is arcwise accessible from two and only two prime ends of the boundary of the unbounded component of $c\left(X_{a}+X_{b}\right)$. Suppose that the two prime ends just identified are 2 and $R$. If $x_{r}$ is a subset of $X_{r}-X$ with $X$ as limit point, one and only one prime end of the unbounded component of $c\left(X_{a}+X_{b}\right)$ is limit of $x_{r}$. Suppose that it is $R ; R$ must contain $X$, must be limit of any subset of $X_{r}-X$ with $X$ as limit point, and must be arcwise accessible from the unbounded connected complementary domain of $X_{a}+X_{b}$. It will be convenient to say that $R$ is limit of $X_{r}$. It may then be proved that 2 and only 2 is limit of each element of $\left[X_{\alpha}\right]$ which is between $X_{a}$ and $X_{b}$, while $R$ and only $R$ is limit of each element of $\left[X_{\alpha}\right]$ which is not between $X_{a}$ and $X_{b}$, prime ends of course of the unbounded component of $c\left(X_{a}+X_{b}\right)$ alone being considered and between being understood with reference to $X_{r}$.

If $X$ is a true continuum, not a point, conditions are somewhat more involved. $X_{a}$ and $X_{b}$ being any two different elements of $\left[X_{\alpha}\right]$, and $\mathcal{C}$ being the simple closed curve of prime ends of the unbounded component of $c\left(X_{a}+X\right.$ $\left.+X_{b}\right)$, then all the elements of $\mathcal{C}$ except possibly exactly four, or exactly three, or exactly two are themselves prime ends of $X_{a}$, or of $X$, or of $X_{b}$. The prime ends of $\mathcal{C}$ not belonging to $X_{a}$ or $X_{b}$ form two components 2 and $R$, and the three possibilities just enumerated respectively characterize the following cases, both 2 and $R$ are true arcs of prime ends of $\mathcal{C}$, either $\mathcal{Q}$ or $R$ is an arc but the other is a single prime end, and both 2 and $R$ are single prime ends. Irrespective of the case considered it can be proved that no element of $\left[X_{\alpha}\right]$ different from $X_{a}$ and $X_{b}$ can have both an end of 2 and an end of $R$ among its limits. Supposing that $R$ contains the limits of $X_{r}$, it can then be shown that $R$ contains all the limits of those elements of $\left[X_{\alpha}\right]$ which are not between $X_{a}$ and $X_{b}$ while 2 contains all the limits of those elements of $\left[X_{\alpha}\right]$ which are between $X_{a}$ and $X_{b}$.

Lемма IV. Given (1) $Z$ is the set defined above and $X$ is a point; (2) 2 is the prime end identified above of the unbounded component of $c\left(X_{a}+X_{b}\right) ;(3) X_{1}$, $X_{2}, \cdots$ is a clockwise series of elements of $\left[X_{\alpha}\right]$ all following both $X_{a}$ and $X_{b}$ and having the prime end 2 as limit; (4) $V$ is a ray in $c\left(X_{a}+X_{r}+X_{b}\right)$ containing a point of $X_{1}$. Then $V$ contains a point of at least one other clement of the series.

Let $V^{*}$ be determined as it was in the proof of Lemma III. Suppose that, for every subscript $n$ greater than $1, X_{n} \cdot V^{*}=0$, that is, suppose the lemma to be false. Let $\left[Q_{i}\right]$ be a chain of cuts defining 2 and $\left[\delta_{i}\right]$ be the corresponding chain of domains. Suppose that $X$ is the only limit point of $\sum Q_{i}$, and that 
$Q_{1}+\delta_{1}$ contains no point of $X_{1}+X_{r}+R$. Let $Q_{1}$ be $Q_{1}^{*}$. As 2 is a limit of $\sum X_{i}$, there is a first element $X_{1}^{*}$ of $\left[X_{i}\right]$ following $X_{1}$, containing a point of $Q_{1}^{*}$, and as 2 is not a limit of $X_{1}^{*}$ there is a first one $Q_{2}^{*}$ of $\left[Q_{i}\right]$ following $Q_{1}^{*}$ containing no point of $X_{1}^{*} . \Lambda \mathrm{s} 2$ is a limit of $\sum X_{i}$, there is a first element $X_{2}^{*}$ of $\left[X_{i}\right]$ following $X_{1}^{*}$ containing a point of $Q_{2}^{*}$, and as 2 is not a limit of $X_{2}^{*}$, also a first one $Q_{3}^{*}$ of $\left[Q_{i}\right]$ following $Q_{2}^{*}$ and containing no point of $X_{2}^{*}$. In general for $n=3,4,5, \cdots$ there is a first element $X_{n}^{*}$ of $\left[X_{i}\right]$ following $X_{n-1}^{*}$ containing a point of $Q_{n}^{*}$, and a first one $Q_{n+1}^{*}$ of $\left[Q_{i}\right]$ following $Q_{n}^{*}$ and containing no point of $X_{n}^{*}$. The two infinite series $\left[X_{i}^{*}\right]$ and $\left[Q_{i}^{*}\right]$ satisfy all the conditions imposed by supposition upon the series $\left[X_{i}\right]$ and $\left[Q_{i}\right]$. For $i=1,2,3, \cdots$, let $S_{i}$ be an arc of $Q_{i}^{*}$ whose non-end points include no points of $X_{a}+X_{i}^{*}$ and whose ends belong to $X_{a}$ and $X_{i}^{*}$ respectively. $S_{i}$ together with the two elements of $\left[X_{\alpha}\right]$ in which its ends lie, for each value of $i$, determines a domain containing $v^{*}$, a point of $V^{*}$, but not containing $V^{*}$. Thus $V^{*} \cdot S_{i} \neq 0$. By the method used in the proof of Lemma III a contradiction similar to the one deduced in it can now be obtained.

TheOREM III. Suppose that $Z$ is a continuum and $X$ a point. If $X_{a}$ and $X_{b}$ are distinct elements of $\left[X_{\alpha}\right]$ and 2 is the prime end of the unbounded connected complementary domain of their sum which contains $X$ and is arcwise accessible from this domain and is not a limit of $X_{r}$, and if $\left[X_{i}\right]$ is a clockwise series of elements of $\left[X_{\alpha}\right]$ all following both $X_{a}$ and $X_{b}$, then 2 is not a limit of $\sum X_{i}$.

Suppose the theorem is not true; suppose in short that the prime end 2 is limit of $\left[X_{i}\right]$. It is clear that all the hypotheses of Lemma IV are now fulfilled or may easily be fulfilled. The process used in obtaining a contradiction to establish Theorem II is now available in this case also.

Corollary III. Suppose $Z$ is a continuum. If $X_{a}$ and $X_{b}$ are distinct elements of $\left[X_{\alpha}\right]$ and 2 is that maximal arc of prime ends of the unbounded component of $c\left(X_{a}+X+X_{b}\right)$ none of which is already a prime end of $X_{a}$ or $X_{b}$ and none of which contains a limit point of $X_{r}-X_{r} \cdot X$, and if $\left[X_{i}\right]$ is a clockwise series of elements of $\left[X_{\alpha}\right]$ all following both $X_{a}$ and $X_{b}$, then no prime end in 2 contains a limit point of $\sum X_{i}$.

Corollary III may be inferred from Theorem III in the way that Corollary II was inferred from Theorem II.

THEOREM IV. If $W$ is a plane bounded continuum composed of the point $X$ and a. set of continua $\left[X_{\alpha}\right]$ no two of which have any point in common except the point $X$ which is common to all, then $W$ separates the point $p$ from the point $q$ 
only if some element of $\left[X_{\alpha}\right]$ separates $p$ from $q . \dagger$

Corollary IV. If $W$ is a plane bounded continuum composed of the continuum $X$ and a set of continua $\left[X_{\alpha}\right]$ each having a point in common with $X$ and no two having in common any point which is not a point of $X$, and, $p$ and $q$ being two distinct points of the complement of $W$ and $X_{a}$ being any element at all of $\left[X_{\alpha}\right]$, if $X+X_{a}$ does not separate $p$ from $q$, then $W$ does not separate $p$ from $q$.

As $X+X_{a}$ does not separate $p$ from $q, X$ does not separate $p$ from $q$ and so owing to the availability of inversion it may as well be assumed that both $p$ and $q$ belongs to $\gamma$, the unbounded component of $c(X)$. In place of $W$ and $X$, regard now the continuum $X^{*}$ which is the sum of $X$ and all its bounded connected complementary domains, and the continuum $W^{*}$ which is the sum of $X^{*}$ and the continua $\left[X_{a}^{*}\right]$, that subset of $\left[X_{\alpha}\right]$ comprising all those elements of $\left[X_{\alpha}\right]$ with points in $\gamma$. Upper semicontinuity now provides a means of reducing the question to the one solved by Theorem IV.

† Since the submission of this paper to the editors a proof of Theorcm IV has appeared in print. Accordingly, although the published demonstration is quite different from the one formerly contained in this paper, it having resembled in its principal details the argument for Theorem II, the proof of the theorem has been omitted. For the proof see J. H. Roberts, Concerning collections of continua not all bounded, American Journal of Mathematics, vol. 52 (1930), pp. 551-562, Theorem I on p. 553.

UNIVERSITY OF TEXAS, Austin, Trixas 